\title{
A Study of the Source Traffic Generator Using Poisson Distribution for ABR Service
}

\author{
Mohsen Hosamo \\ Department of Computer Engineering, Wadi International University, The Valley, Homs, Syria \\ Correspondence should be addressed to Mohsen Hosamo, mohsen_hosamo@yahoo.com \\ Received 8 April 2012; Accepted 18 June 2012 \\ Academic Editor: Agostino Bruzzone
}

Copyright () 2012 Mohsen Hosamo. This is an open access article distributed under the Creative Commons Attribution License, which permits unrestricted use, distribution, and reproduction in any medium, provided the original work is properly cited.

This paper describes modeling of the available bit rate (ABR) source traffic in asynchronous transfer mode (ATM) network using $\mathrm{BL}_{\mathrm{Pos}} / \mathrm{GT}_{\mathrm{EXP}}$ traffic generator, which employs Poisson distribution for modeling the burst length (BL $\mathrm{L}_{\mathrm{Pos}}$ ) and exponential distribution for modeling the gap time $\left(\mathrm{GT}_{\mathrm{EXP}}\right)$. This traffic generator inherits the advantages of both Poisson and exponential distribution functions to achieve enhanced link performance. Analytical and simulation results for BLPos $/ \mathrm{GT}_{\mathrm{EXP}}$ traffic generator have been presented and compared.

\section{Introduction}

The Poisson process is an extremely useful process for modeling purposes in many practical applications, such as, for example, to model arrival processes for queueing models or demand processes for inventory systems. It is empirically found that in many circumstances the arising stochastic processes can be well approximated by a Poisson process. From the standpoint of the network users, the messages within a session are typically triggered by particular events. But from the standpoint of the network, these message initiations are somewhat arbitrary and unpredictable. Therefore, the sequence of times at which messages arrive for a given session is a random process. Further, the arrival process of one session has to be independent of others. The poisson process has been proved to be the most appropriate for such purpose and therefore has been considered in the present work.

In this paper source traffic modeling and simulation have been carried out for ABR service in ATM networks using Poisson distribution for modeling the burst length (BLPos) and exponential distribution for modeling the gap time $\left(\mathrm{GT}_{\mathrm{Exp}}\right)$. The corresponding traffic generator is designated as $\mathrm{BL}_{\mathrm{Pos}} / \mathrm{GT}_{\mathrm{Exp}}$.

\section{2. $\mathrm{BL}_{\mathrm{Pos}} / \mathrm{GT}_{\mathrm{EXP}}$ Traffic Generator}

$\mathrm{BL}_{\mathrm{Pos}} / \mathrm{GT}_{\mathrm{EXP}}$ traffic generator generates cells sent at a fixed rate (ACR) during BL and no cells are sent during GT. $\mathrm{BL}$ is assumed to be Poisson distributed ( $\mathrm{BL}_{\mathrm{Pos}}$ ) whereas GT is exponentially distributed $\left(\mathrm{GT}_{\mathrm{Exp}}\right)$. $\mathrm{BL}_{\mathrm{Pos}} / \mathrm{GT}_{\mathrm{EXP}}$ traffic generator was created and parameterized as explained below.

When events occur according to an exponential distribution, they are said to occur completely at random. Thus the arrival time of the next event is not affected by the time elapsed since the previous event [1-5]. An exponential random variable with a mean value of $\lambda_{\operatorname{Exp}}$ (where $\lambda_{\operatorname{Exp}}>0$ ) is given by the following probability density function (PDF):

$$
f(x)_{\operatorname{Exp}}=\left\{\begin{array}{cc}
\lambda_{\operatorname{Exp}} \cdot \mathrm{e}^{-\lambda_{\mathrm{Exp}} \cdot x} & x \geq 0 \\
0 & x<0
\end{array}\right\},
$$

and the cumulative density function (probability that time between events is $x$ ) can be obtained by integrating (1):

$$
F(x)_{\operatorname{Exp}}=\left\{\begin{array}{cc}
1-\mathrm{e}^{-\lambda_{\operatorname{Exp}} \cdot x} & x \geq 0 \\
0 & x<0
\end{array}\right\} .
$$


The mean and variance of the exponential distribution are

$$
\begin{gathered}
E(x)_{\operatorname{Exp}}=\int_{0}^{\infty} x \cdot \lambda_{\operatorname{Exp}} \cdot e^{-\lambda_{\operatorname{Exp}} \cdot x} d x=\frac{1}{\lambda_{\operatorname{Exp}}}, \\
\operatorname{Var}(x)_{\operatorname{Exp}}=\int_{0}^{\infty} x^{2} \cdot \lambda_{\operatorname{Exp}} \cdot e^{-\lambda_{\operatorname{Exp}} \cdot x} d x-\left(\frac{1}{\lambda_{\operatorname{Exp}}}\right)^{2}=\frac{1}{\lambda_{\operatorname{Exp}}^{2}} .
\end{gathered}
$$

A Poisson random variable $x$ with parameter $\mu_{\text {Pos }}$ (where $\mu_{\text {Pos }}>0$ ) has PDF given by $[1,2]$

$$
f(x)_{\mathrm{Pos}}=\frac{\mu_{\mathrm{Pos}}^{x}}{x !} e^{-\mu_{\mathrm{Pos}}}, \quad x=0,1,2, \ldots
$$

The mean and variance are identical for the Poisson distribution [1]:

$$
E(X)_{\mathrm{Pos}}=\operatorname{Var}(X)_{\mathrm{Pos}}=\mu_{\mathrm{Pos}},
$$

where a Poisson distribution can be developed by involving the definition of a Poisson Process for a fixed time $(t)$, so that $\mu_{\text {Pos }}=\lambda_{\text {Exp }} \cdot t$. For Poisson distributed events, the time between successive events is exponentially distributed with mean $1 / \mu_{\text {Pos }}$. For an event that occurs at a time interval with an exponential distribution, the rate of occurrence of the event is Poisson distributed with mean $\lambda_{\text {Exp }}$. Required distribution modeling involves a transformation function for converting a random variable of uniform distribution into the required distribution. Considering the fundamental transformation law of probabilities for two probability density functions $f(x)$ and $p(u)$

$$
|f(x) d x|=|p(u) d u| \quad \text { or } \quad f(x)=p(u)\left|\frac{d u}{d x}\right|,
$$

where $p(u)$ is the PDF of random variable $u$ and $f(x)$ is another PDF of random variable $x$. Since $u$ is a random variable of a uniform distribution in the range 0 to $1, p(u)$ is a constant $(=1)$ and hence

$$
f(x)=\frac{d u}{d x} \quad \text { and therefore } u=F(x)=\int_{0}^{x} f(z) d z .
$$

Equation (7) can be used to find source random variable $x=$ $G(u)$ through inverse transformation of $u=F(x)$. For the required distribution, the inverse can easily be found from (7) with $f(z)$ corresponding to the required distribution. $u$ is uniformly distributed in the range $0 \leq u \leq 1$. It can be generated by using the function rand() provided by the standard Linux library or using Mersenne Twister (MT) [6].

For modeling the $\mathrm{GT}_{\mathrm{Exp}}$, (7) is used with

$$
u=F(x)=1-e^{-\lambda_{\operatorname{Exp}} \cdot x} \quad \text { or } \quad 1-u=e^{-\lambda_{\operatorname{Exp}} \cdot x} .
$$

Therefore, the required transformation is

$$
x=-\frac{1}{\lambda_{\operatorname{Exp}}} \cdot \ln (1-u) .
$$

By changing the variables $x$ and $(1-u)$ of (9) with $X_{\operatorname{Exp}}$ and $U$ respectively, we get

$$
X_{\operatorname{Exp}}=-\frac{1}{\lambda_{\operatorname{Exp}}} \cdot \ln (U) .
$$

2.1. Estimation of the Load $\left(L_{i}\right)$ for the Traffic Generator. The load variation of the traffic can be realized by synthesizing predefined load such that the resulting load $L=\sum_{i=1}^{N} L_{i}$, where $L_{i}$ is the traffic load due to $i$ th source. Therefore, the aggregate traffic from $N$ sources will generate the load $L$ on a link with rate $R$ Mbps giving average throughput of $R \cdot L$ Mbps. The load $L_{i}$ generated by an individual source can be expressed as

$$
L_{i}=\frac{\overline{\mathrm{BL}_{\mathrm{Pos}}} \cdot K}{\overline{\mathrm{BL}_{\mathrm{Pos}}} \cdot\left(K+P_{r}\right)+\overline{\mathrm{GL}_{\mathrm{Exp}}}},
$$

where $\overline{\mathrm{BL}_{\text {Pos }}}, \overline{\mathrm{GL}_{\mathrm{Exp}}}, K$, and $P_{r}$ are the mean $\mathrm{BL}_{\mathrm{Pos}}$, mean gap length, cell size, and minimum intercell gap length (Preamble), respectively in bytes, and then the load $L_{i}$ can be found from (11).

2.2. Estimation of the Minimum Gap Time $\left(M_{G T_{E x p}}\right)$. The minimum $\mathrm{GT}_{\mathrm{Exp}}\left(M_{\mathrm{GT}}\right)$ is a secondary parameter dependent on load. Given a desired load, $M_{\mathrm{GT}}$ Exp is calculated by the source automatically using exponential distribution. Using (11) the $\overline{\mathrm{GT}_{\mathrm{Exp}}}$ can be expressed as

$$
\overline{\mathrm{GL}_{\mathrm{Exp}}}=\overline{\mathrm{BL}} \cdot\left[K \cdot \frac{1-L_{i}}{L_{i}}-P_{r}\right] .
$$

The $\overline{\mathrm{BL}_{\text {Pos }}}$ can be written as

$$
\overline{\mathrm{BL}_{\mathrm{Pos}}}=E(x)_{\mathrm{BL}_{\mathrm{Pos}}}=M_{\mathrm{BL}} \text {, }
$$

where $M_{\mathrm{BL}}$ is the minimum BLPos.

$\overline{\mathrm{GL}_{\mathrm{Exp}}}$ can be written as

$$
\overline{\mathrm{GL}_{\mathrm{Exp}}}=E(x)_{\mathrm{GL}_{\mathrm{Exp}}}=M_{\mathrm{GL}} .
$$

Substituting the values of $\overline{\mathrm{BL}_{\text {Pos }}}$ and $\overline{\mathrm{GL}_{\text {Exp }}}$ from (13) and (14), respectively, in (12) $M_{\mathrm{GL}_{\mathrm{Exp}}}$ can be written as

$$
M_{\mathrm{GL}}=M_{\mathrm{BLp}} \cdot\left[\frac{K}{L_{i}}-\left[K+P_{r}\right]\right] .
$$

Considering the link rate and using the following relation:

$$
\text { Byte Time }=\frac{\text { Byte Size }(\text { bits })}{\text { Link Rate }(\text { bits } / \text { sec })} \quad \text { or } \quad \text { Byte Time }=\frac{b}{R} \text {, }
$$

the $M_{\mathrm{GT}}$ now can be computed as

$$
M_{\mathrm{GT}_{\mathrm{Exp}}}=\frac{b}{R} \cdot M_{\mathrm{BL}_{\mathrm{Pos}}} \cdot\left[\frac{K}{L_{i}}-\left[K+P_{r}\right]\right] .
$$

Now the value of $P_{r}=1 /$ ACR is readily available, depending upon the selected value(s) of ACR that can be separately taken as variable, and thus (17) can be rewritten as

$$
M_{\mathrm{GT}_{\mathrm{Exp}}}=\frac{K \cdot b}{R} M_{\mathrm{BL} \mathrm{Pos}_{\mathrm{p}}}\left[\frac{1}{L_{i}}-1\right]
$$

Therefore, (18) can be used for computing the value of $M_{\mathrm{GT}} \mathrm{Exp}_{\mathrm{Ex}}$ that would result in link load closer to $L_{i}$ using 
the selected values of $L_{i}$ of the $i$ th source, $K$, and Poisson distribution parameters. Considering the same parameter values for burst and gap lengths and $M_{\mathrm{BL}_{\mathrm{Pos}}}=1$, (18) can be simplified as

$$
M_{\mathrm{GT}_{\mathrm{Exp}}}=\frac{K \cdot b}{R} M_{\mathrm{BL} \mathrm{Pos}}\left[\frac{1}{L_{i}}-1\right] .
$$

2.3. Generating the $G T_{\mathrm{EXP}}$. Bearing in mind that $\lambda_{\mathrm{Exp}}=\mathrm{ACR}$, since in $A B R$ service a source sends its data with a rate equal to ACR, defining $\lambda_{\mathrm{GT}_{\mathrm{Exp}}}$ as the exponential mean arrival rate for $\mathrm{GT}_{\mathrm{Exp}}$, and considering (14) in terms of time, we get (20)

$$
E(x)_{\mathrm{GT}_{\mathrm{Exp}}}=\frac{1}{\lambda_{\mathrm{GT}_{\mathrm{Exp}}}}=M_{\mathrm{GT}_{\mathrm{Exp}}} .
$$

Now the $\mathrm{BL}_{\mathrm{Pos}} / \mathrm{GT}_{\mathrm{EXP}}$ traffic generator can compute the $\mathrm{GT}_{\text {Exp }}$ using the relation

$$
\mathrm{GT}_{\mathrm{Exp}}=-M_{\mathrm{GT}_{\mathrm{Exp}}} \cdot \ln (U) .
$$

2.4. BL $L_{\text {Pos }}$ Generating. BLPos can be modeled as follows: for ABR service in ATM networks the value of ACR should be within the range $\mathrm{MCR} \leq \mathrm{ACR} \leq \mathrm{PCR}$. The source first starts transmitting a random-sized burst of cell at ICR. It then waits for a random amount of time, which follows exponential distribution. The source will go on repeating this cycle $\mathrm{BL}_{\mathrm{Pos}} / \mathrm{GT}_{\mathrm{Exp}}$ until completing transmission. In every cycle the number of cells is determined by ACR, which is changed due to the feedback used in the switch. The source repeats the calculation of Poisson distribution, (4) with $\mu_{\text {Pos }}=A C R$, $k$ times, where $k$ is changing from 0 to peak cell rate (PCR). The results will be saved in an array with size $k$. The number of cells inside the burst can be found by generating a random number $u$ [6], applying the cumulative distribution function (cdf) for all array values, so that the cumulative values are smaller than or equal to a random number $u$. The following relation gives the $\mathrm{BL}_{\mathrm{Pos}}$ that is used by the $\mathrm{BL}_{\mathrm{Pos}} / \mathrm{GT}_{\mathrm{Exp}}$ traffic generator:

$$
\mathrm{BL}_{\mathrm{Pos}}=\sum_{x=0}^{x=u}\left(e^{-\mu_{\mathrm{Pos}}} \cdot \frac{\mu_{\mathrm{Pos}}^{-x}}{x !}\right), \quad \mu_{\mathrm{Pos}}=\mathrm{ACR},
$$

where the number of cells inside the burst should be at least one $\left(M_{\mathrm{BL}}=1\right)$.

\section{Analytical Results}

Consider the values of $L_{i}, R, K, M_{\mathrm{BL} \text { os }}$, Poisson mean arrival rate $\left(\mu_{\mathrm{BL}_{\mathrm{Pos}}}\right)$, and $\lambda_{\mathrm{GT}_{\mathrm{Exp}}}$ for $\mathrm{BL}_{\mathrm{Pos}} / \mathrm{GT}_{\mathrm{Exp}}$ traffic generator as given in Table 1 . The value of $M_{\mathrm{GT}}$ Exp was determined using (19) (Table 1).

The analytical result of $\mathrm{BL}_{\mathrm{Pos}} / \mathrm{GT}_{\mathrm{Exp}}$ traffic generator for 1000 count values of $U$, generated by the uniform distribution, are shown in Figure 1, and the corresponding computed values of mean, variance, maximum, and minimum values of BL and GT, and are given in Table 2.

The variations in $\mathrm{BL}_{\mathrm{Pos}}, \mathrm{GT}_{\mathrm{Exp}}$ as functions of $\mu_{\mathrm{BL}}$ and $\lambda_{\mathrm{GT}_{\mathrm{Exp}}}$ for $\mathrm{BL}_{\mathrm{Pos}} / \mathrm{GT}_{\mathrm{Exp}}$ traffic generator for 100 count
TABLE 1: The evaluated parameters for the $\mathrm{BL}_{\mathrm{Pos}} / \mathrm{GT}_{\mathrm{Exp}}$ traffic generator.

\begin{tabular}{lc}
\hline The parameters & The values \\
\hline$L_{i}$ & 0.3 \\
$R$ & $149.76 \mathrm{Mbps}$ \\
$K$ & $53 \cdot 8 \mathrm{bits}$ \\
$M_{\mathrm{BLPos}}$ & $1 \mathrm{cell}$ \\
$\mu_{\mathrm{BL} \text { Pos }}$ & $1 \mathrm{cell} / \mathrm{sec}$ \\
$\lambda_{\mathrm{GT}_{\mathrm{Exp}}}$ & $30 \mathrm{cells} / \mathrm{sec}$ \\
$M_{\mathrm{GT}}$ & $0.2202 \mu \mathrm{sec}$ \\
\hline
\end{tabular}

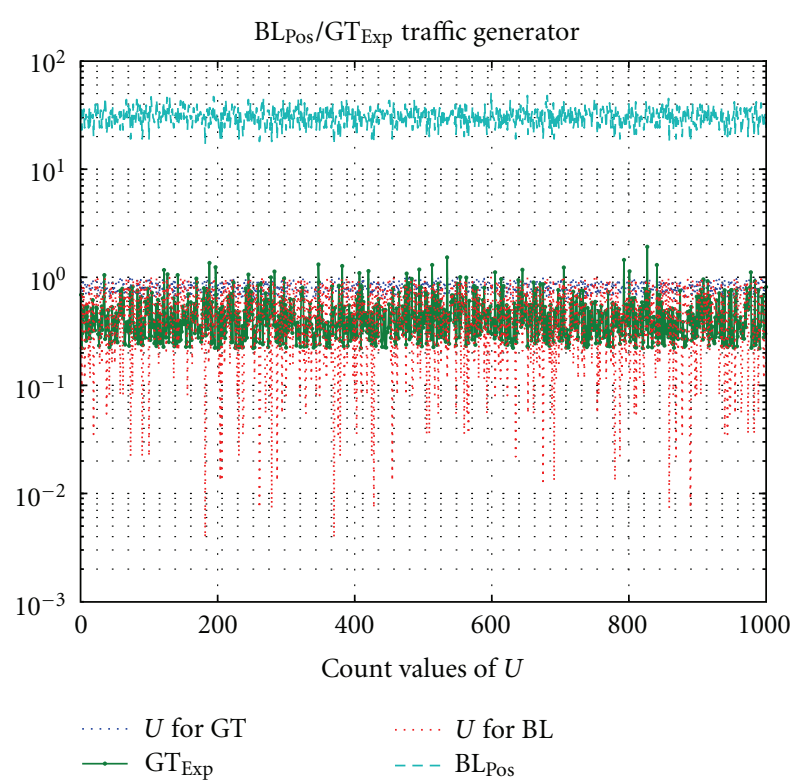

FIGURE 1: $\mathrm{BL}_{\text {Pos }} / \mathrm{GT}_{\text {Exp }}$ traffic generator for 1000 count values of $U$.

TABLe 2: $\mathrm{BL}_{\mathrm{Pos}}$ (cells) and $\mathrm{GT}_{\mathrm{Exp}}(\mu \mathrm{sec})$ for $\mathrm{BL}_{\mathrm{Pos}} / \mathrm{GT}_{\mathrm{Exp}}$ traffic generators.

\begin{tabular}{lcccc}
\hline & Mean & Variance & Maximum & Minimum \\
\hline BL $_{\text {Pos }}$ & 29.98100 & 31.05969 & 50.00000 & 16.00000 \\
GT $_{\text {Exp }}$ & 0.436102 & 0.043788 & 1.917750 & 0.220461 \\
\hline
\end{tabular}

values of $U$ are shown in Figures 2 and 3 respectively. The increment steps for $\lambda_{\mathrm{BL}_{\mathrm{Pos}}}(1-110)$ cells/sec and $\mu_{\mathrm{GT}_{\mathrm{Exp}}}(1-110)$ cells/sec are 10 for each. Referring to Table 2 it is seen that the minimum values of $\mathrm{BL}_{\mathrm{Pos}} / \mathrm{GT}_{\mathrm{Exp}}$ are greater than their corresponding values of $M_{\mathrm{BL} \text { pos }} / M_{\mathrm{BL}}$. Referring to Figure 2 it can be concluded that the Poisson mean arrival parameter $\mu_{\mathrm{BL}}$ should not be a very large value, because BL $L_{\text {Pos }}$ will, consequently, be very large as well, and the source will spend most of its time sending only the burst cells with a smaller number of gap intervals for $\mathrm{BL}_{\mathrm{Pos}} / \mathrm{GT}_{\mathrm{Exp}}$ traffic generator resulting in less-bursty traffic. Referring to Figure 3 it can be concluded that the exponential mean arrival parameter $\lambda_{\mathrm{GT}_{\mathrm{Exo}}}$ should be selected between 2 and 30 cells/sec for simulation of real bursty traffic because it offers higher peak values of $\mathrm{GT}_{\mathrm{Exp}}$. This is further supported by the observation that for 


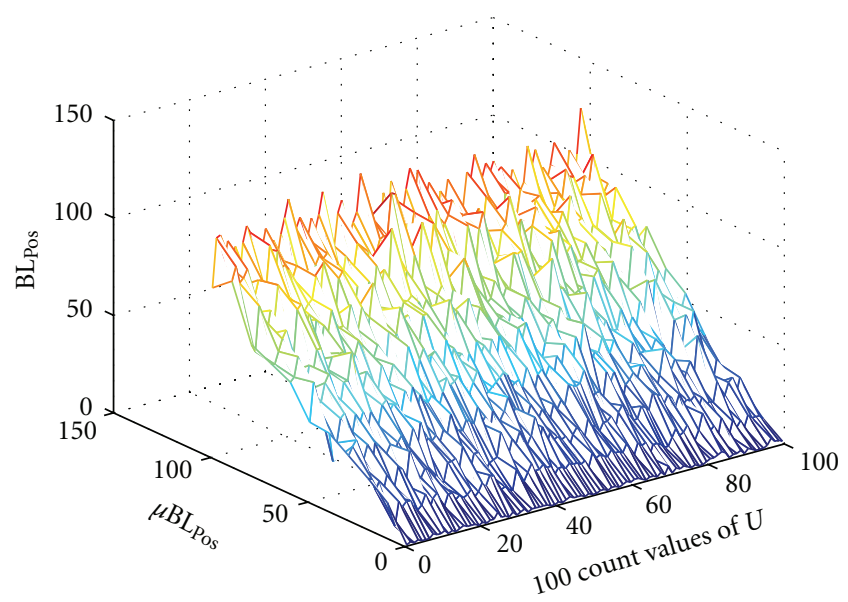

Figure 2: $\mathrm{BL}_{\mathrm{Pos}}$ versus $\mu_{\mathrm{BL} \text { pos }}$ and $U$.

TABLE 3: The values of the ACR (cells/sec).

\begin{tabular}{lcccccc}
\hline & ACR1 & ACR2 & ACR3 & ACR4 & ACR5 & ACR6 \\
\hline Mean & 69.57 & 187.05 & 329.01 & 172.78 & 262.24 & 339.74 \\
Variance & 911.5 & 10334. & 11456. & 42343. & 42850. & 65698. \\
Maximum & 125.28 & 300.00 & 400.00 & 500.00 & 600.00 & 700.00 \\
Minimum & 25.36 & 21.75 & 24.51 & 5.00 & 8.71 & 5.00 \\
\hline
\end{tabular}

TABLE 4: The values of the SWIR, SWOR, MAT, and Q.

\begin{tabular}{lcccc}
\hline & Mean & Variance & Maximum & Minimum \\
\hline SWIR (cells/sec) & 1812.55 & 39712.45 & 2303.96 & 1461.94 \\
SWOR (cells/sec) & 1804.66 & 13421.52 & 2338.33 & 1576.5 \\
MAT (sec) & 0.32 & 0.03 & 0.76 & 0.1 \\
$Q$ & 163.65 & 16902.05 & 618 & 25 \\
\hline
\end{tabular}

$\lambda_{\mathrm{GT}_{\mathrm{Exo}}}$ in the range 30 to 100 cells/sec, the peak values of $\mathrm{GT}_{\mathrm{Exp}}$ have the least variation indicating smoothest traffic.

\section{Simulation Results}

The ATM network simulation was carried out under Linux network programming. The parameters specified in Table 1 were used for this simulation. Six sources $S_{i}(i=1,2, \ldots 6)$ sending their data at the rate $\mathrm{ACR}_{i}(i=1,2, \ldots 6)$ between minimum cell rate (MCR) and peak cell rate (PCR) were considered. The performance of the relative rate marking (RRM) switch was evaluated for traffic generator with respect to the allowed cell rate (ACR), switch input rate (SWIR)/switch output rate (SWOR), memory access time (MAT), queue length $(Q)$, and cell transfer delay (CTD). The initial value of ACR for sources $S_{i}$ was taken as PCR/2 whereas the final ACR value was kept between 200 to 700 cells/sec in incremental steps of 100 for $i=1,2, \ldots 6$ and taking buffer size $=1000$ cells, higher queue threshold $\left(Q_{H}\right)=200$ cells, lower queue threshold $\left(Q_{L}\right)=100$ cells, and assuming that each source has to send a total of 1000 cells. The variations in ACR, SWIR/SWOR, MAT, $Q$, and CTD as a function of time are given, respectively, in Figures 4-8.

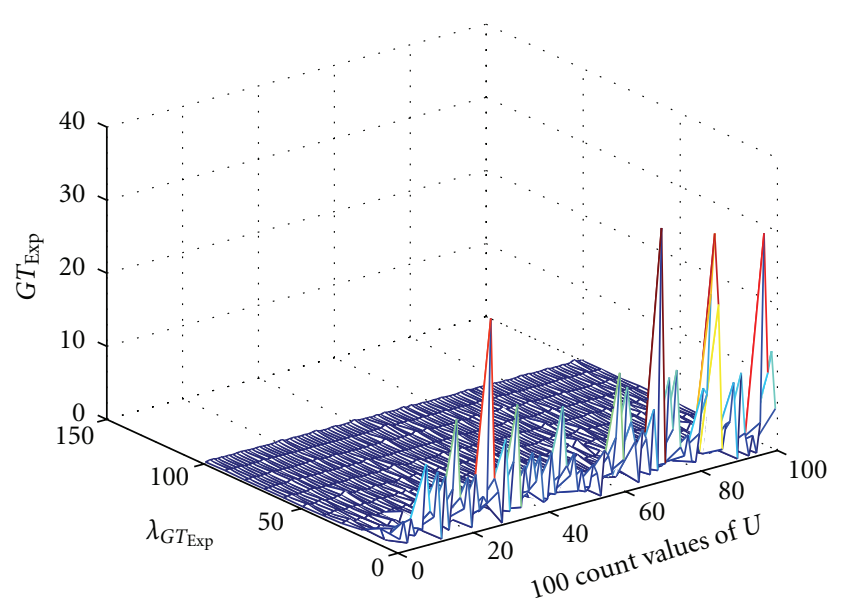

Figure 3: $\mathrm{GT}_{\mathrm{Exp}}$ versus $\lambda_{\mathrm{GT}_{\mathrm{Exo}}}$ and $U$.

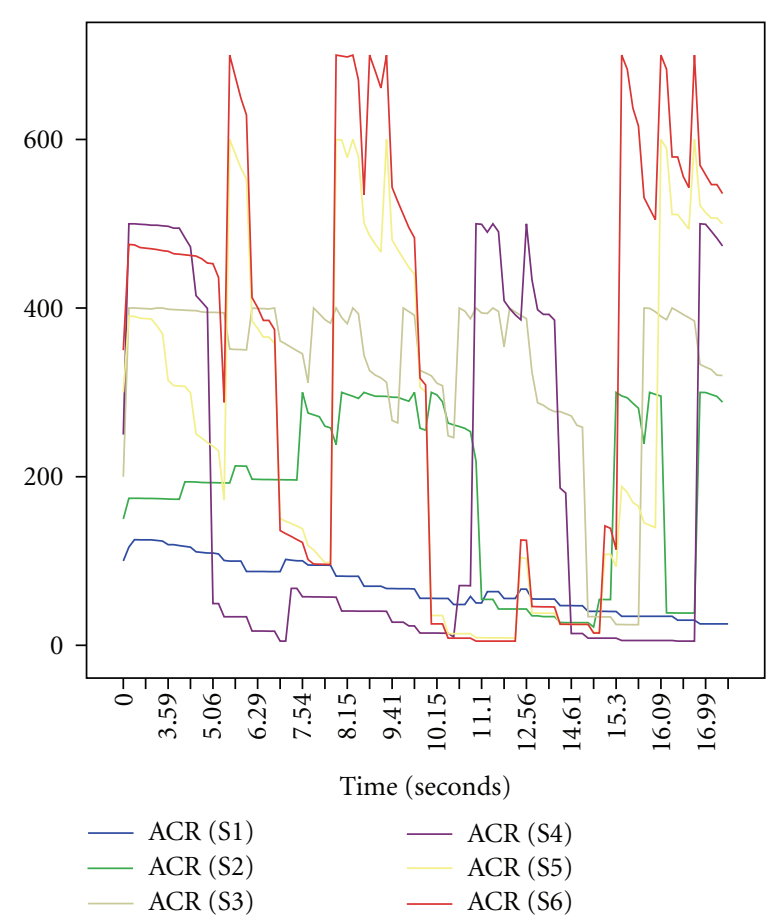

FIGURE 4: The variation of ACR.

The simulation results for mean, variance, maximum and minimum values of ACR, SWIR, SWOR, MAT, $Q$ and CTD are given in Tables 3, 4 , and 5 respectively.

It can be seen from Figure 4 that ACR changes due to the feed back received from the switch. When the switch is heavily loaded (congestion) there is a decrease in the ACR and when it is lightly loaded (no impeding congestion) the ACR is either kept constant or it is increased. The relative changes between the SWIR and SWOR are shown in Figure 5 are responsible for the variations in the MAT (Figure 6) and $Q$ (Figure 7). When the SWIR becomes greater than SWOR the buffer starts filling and reaches a specified threshold level. The switch then signals the source to start reducing its data rate. Consequently, source ACR reduces, and its effect 


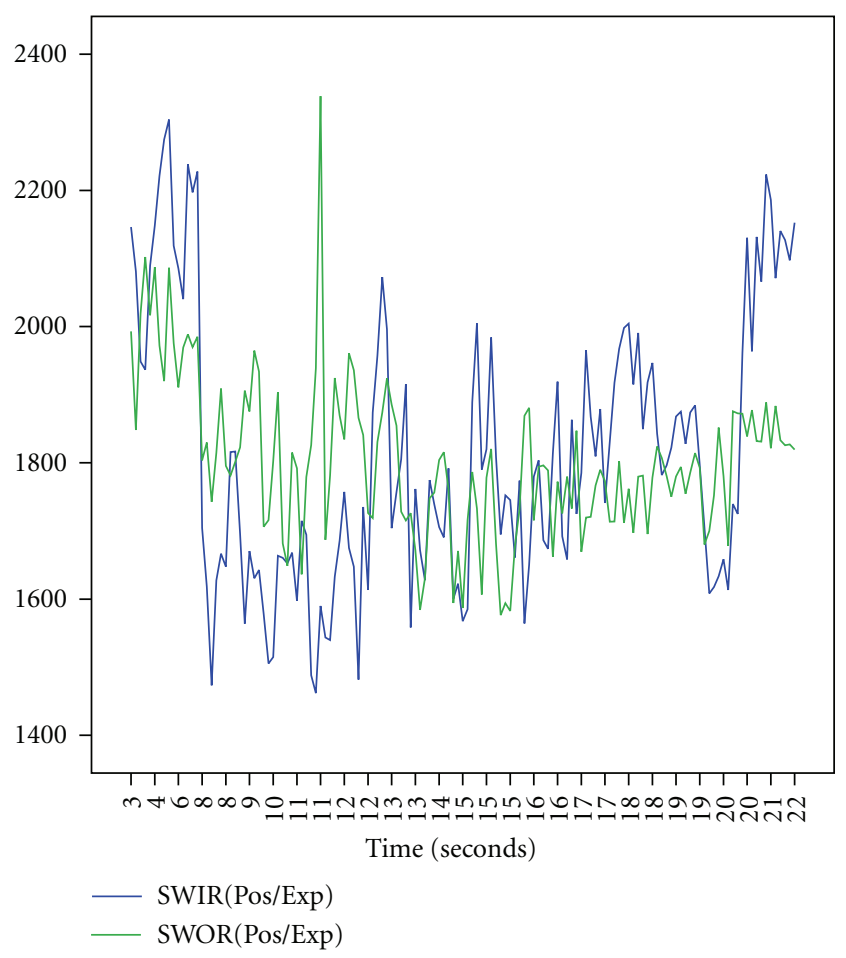

FIgURE 5: The variation of SWIR/SWOR.

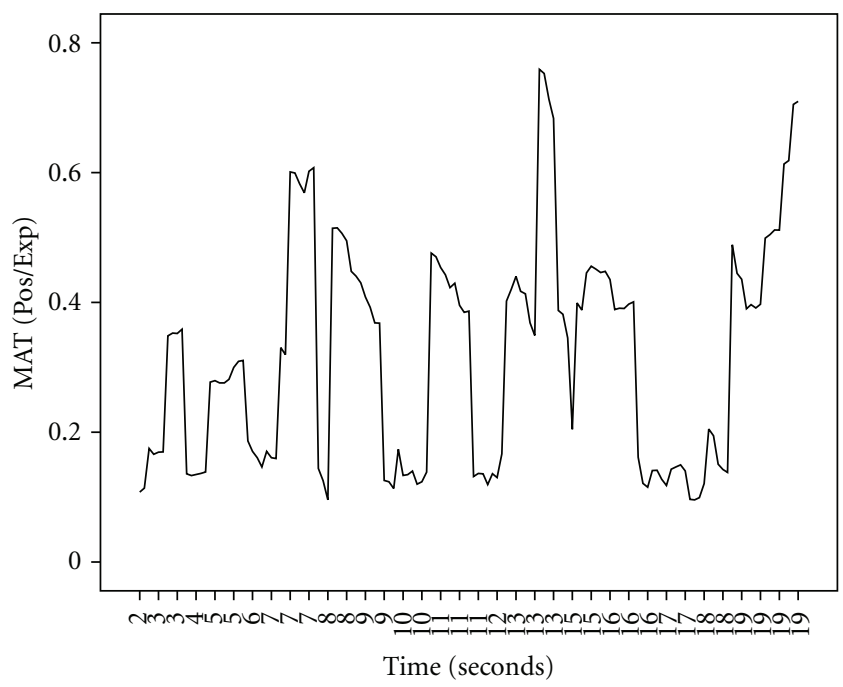

FIgure 6: The variation of MAT.

appears at the queue, which causes a reduction in the rate of increase in the queue size. For a SWIR smaller than a SWOR, the buffer starts becoming empty and when $Q$ reaches its minimum value, the source is signaled to start increasing its data rate. There is a time lag between the switch experiencing a traffic load variation, effect of switch feedback control, and the occurrence of the new load due to the feedback.

Referring to Figure 8, and Table 5 it is noticed that the CTD between any source and its corresponding destination changes from minimum value of $0.2 \mathrm{sec}$ to a maximum value of $1.04 \mathrm{sec}$.

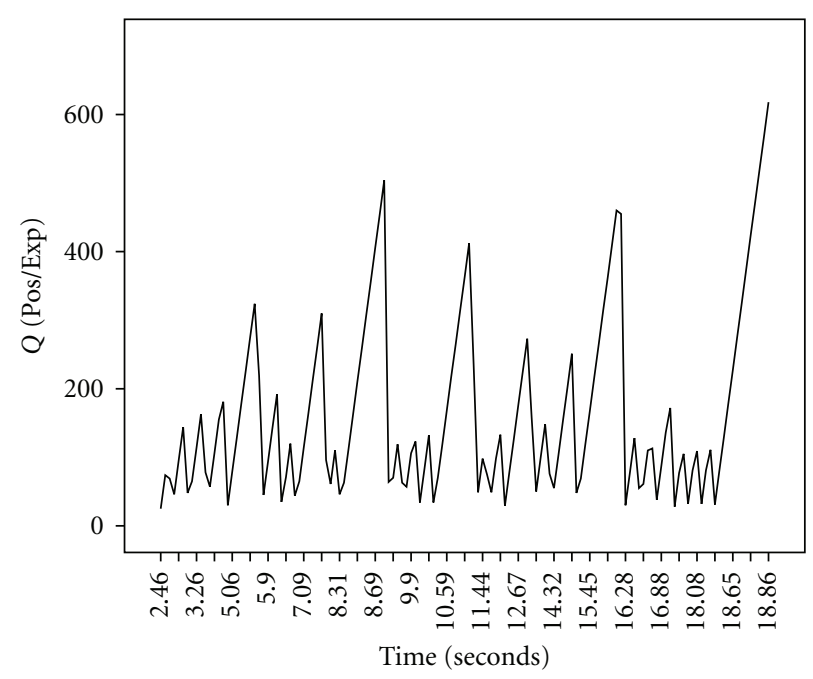

FIgURE 7: The variation of $Q$.

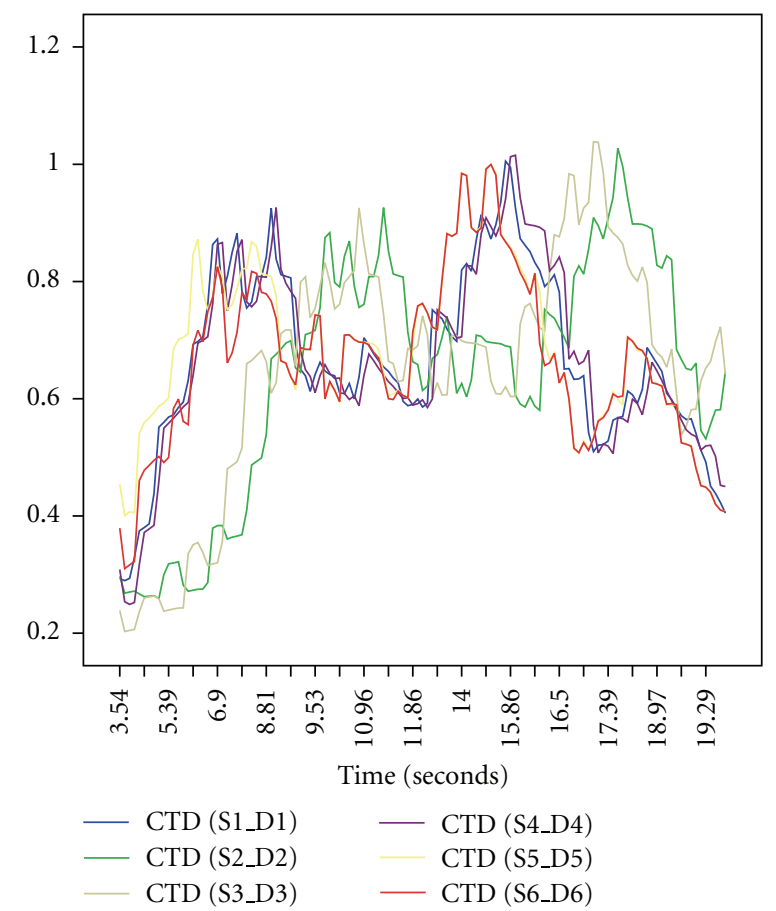

FIgURE 8: CTD using $\mathrm{BL}_{\mathrm{Pos}} / \mathrm{GT}_{\mathrm{Exp}}$ traffic generator.

Examining the simulation results for ACR, SWIR, SWOR, MAT, $Q$ and CTD, which indicate the performance of RRM switch under $\mathrm{BL}_{\text {Pos }} / \mathrm{GT}_{\text {Exp }}$ traffic generator, it is seen that the switch offers the best performance.

\section{Conclusion}

In this paper a mathematical modeling of the $\mathrm{BL}_{\mathrm{Pos}} / \mathrm{GT}_{\operatorname{Exp}}$ source traffic generator has been carried out. The analytical results showed that the minimum values of $\mathrm{BL}_{\mathrm{Pos}} / \mathrm{GT}_{\operatorname{Exp}}$ are greater than their corresponding values of $\mathrm{M}_{\mathrm{BL}_{\mathrm{Pos}}} / \mathrm{M}_{\mathrm{BL}_{\mathrm{Exp}}}$ and the Poisson mean arrival parameter $\mu_{\mathrm{BL}_{\mathrm{Exp}}}$ should not 
TABLE 5: The values of the CTD (sec).

\begin{tabular}{lcccccc}
\hline & CTD (S1_D1) & CTD (S2_D2) & CTD (S3_D3) & CTD (S4_D4) & CTD (S5_D5) & CTD (S6_D6) \\
\hline Mean & 0.67 & 0.64 & 0.65 & 0.67 & 0.69 & 0.67 \\
Variance & 0.02 & 0.04 & 0.04 & 0.03 & 0.02 & 0.02 \\
Maximum & 1.01 & 1.03 & 1.04 & 0.25 & 1 & 0.4 \\
Minimum & 0.29 & 0.26 & 0.2 & 0.25 & 0.31 \\
\hline
\end{tabular}

be a very large value, because $\mathrm{BL}_{\mathrm{Pos}}$ will, consequently, be very large as well, and the source will spend most of its time sending only the burst cells with a smaller number of gap intervals for $\mathrm{BL}_{\mathrm{Pos}} / \mathrm{GT}_{\mathrm{Exp}}$ traffic generator resulting in less-bursty traffic. It can be concluded also that the exponential mean arrival parameter $\lambda_{\mathrm{GT}_{\mathrm{Exo}}}$ should be selected between 2 and 30 cells/sec for simulation of real bursty traffic because it offers higher peak values of $\mathrm{GT}_{\mathrm{Exp}}$. This is further supported by the observation that for $\lambda_{\mathrm{GT}_{\mathrm{Exo}}}$ in the range 30 to 100 cells/sec, the peak values of $\mathrm{GT}_{\mathrm{Exp}}$ have the least variation indicating smoothest traffic. The analytical results have been verified through simulation for six sources.

\section{References}

[1] H. William and M. Douglas, Probability Statistics in Engineering and Management Science, John Wiley and Sons, New York, NY, USA, 3rd edition, 1990.

[2] T. Dias, W. Keerthipala, and I. Murray, "Performance analysis of Aloha channels with self similar input traffic," in Proceedings of the 11th Asian Test Symposium (ATS '02), pp. 255-262, San Diego, Calif, USA, 2000.

[3] M. Ghanbari, C. Hughes, M. Sinclair, and J. Eade, Principles of Performance Engineering for Telecommunication and Information Systems, The Institute of Electrical Engineers, London, UK, 1997.

[4] A. Leon-Garcia, Probability and Random Processes for Electrical Engineering, Addison Wesley, Reading, Mass, USA, 2nd edition, 1994.

[5] M. Sadiku and M. Ilyas, Simulation of Local Area Networks, CRC Press, Florida, Fla, USA, 1995.

[6] M. Matsumoto and T. Nishimura, "Mersenne twister: a 623dimensionally equidistributed uniform pseudo-random number generator," ACM Transactions on Modeling and Computer Simulation, vol. 8, no. 1, pp. 3-30, 1998. 

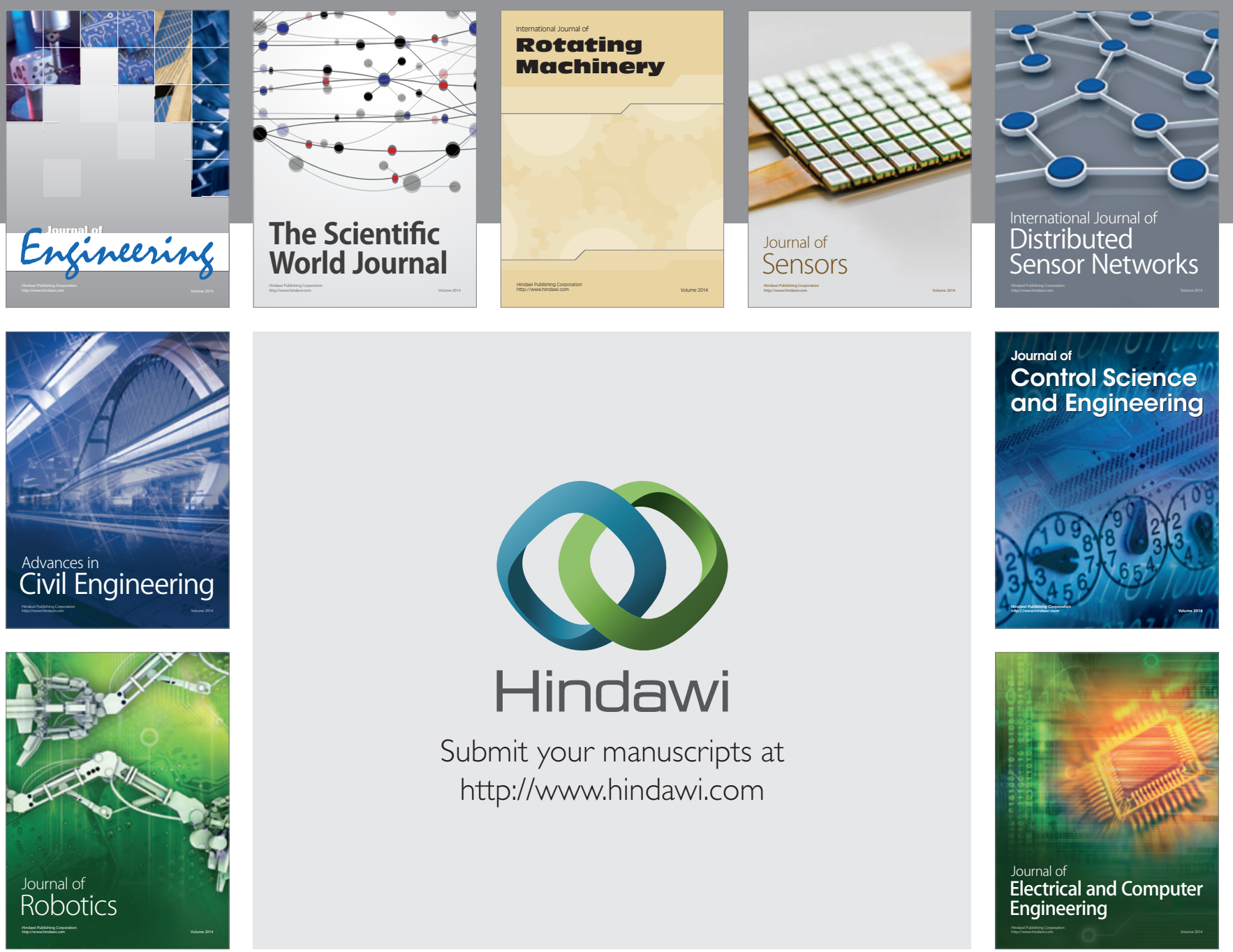

Submit your manuscripts at

http://www.hindawi.com
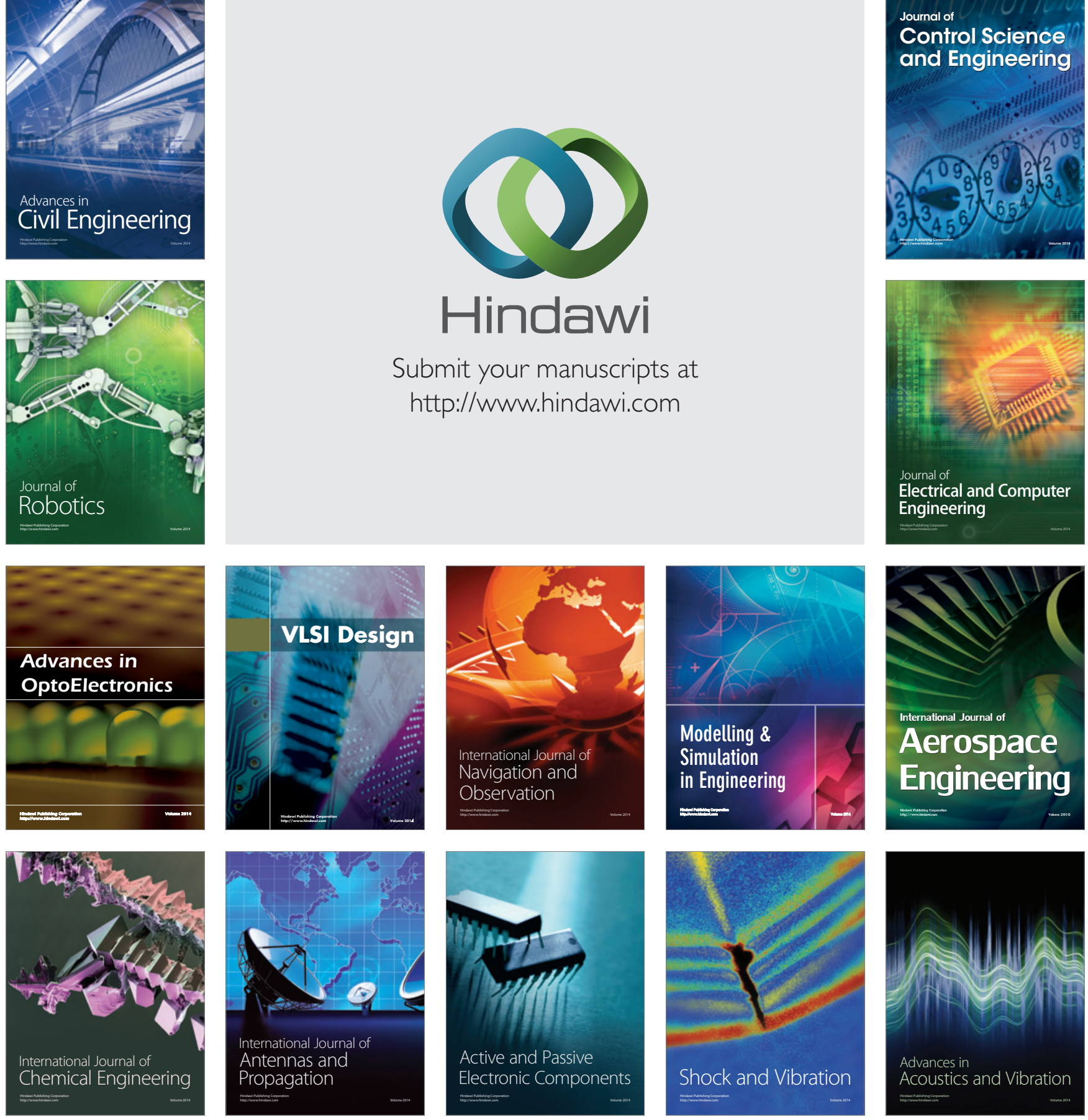